\title{
Effect of a Thermal Nonlinear Absorption Coefficient on the Dynamics of a Photovoltaic Panel
}

\author{
Konga Eric Kapim Abraham Pelap François * \\ UR de Mécanique et de Modélisation des Systèmes Physiques (UR-2MSP), Faculté des Sciences, Université de \\ Dschang, BP 69 Dschang, Cameroun
}

\begin{abstract}
In this article, we propose a theoretical study of the effect of a thermal nonlinear absorption coefficient on the dynamics of a photovoltaic (PV) panel. This coefficient is expressed as a quadratic function of temperature of a PV module known as active zone of a PV panel. Based on the energy balance at each layer of a PV panel, the mathematical expressions of the temperature on each layer are derived. A comparative study of the thermal behavior of a PV panel for a constant and nonlinear coefficient is made, thanks to the numerical investigations carried out in the MATLAB environment. We reveal that, this nonlinear absorption coefficient induces the cooling of a PV panel as a whole, and optimize the electrical efficiency of a PV panel around $2.5 \%$.
\end{abstract}

Keywords: Nonlinear absorption coefficient, recombination process, electrical efficiency, diffusion process, PV panel.

DOI: $10.7176 / \mathrm{JETP} / 10-6-02$

Publication date:October $31^{\text {st }} 2020$

\section{Introduction}

Energy production today, is a huge challenge. Indeed, as the energy needs of industrialized countries increase exponentially over time, they need more energy to develop their countries. Today, most of the world's energy production is provided by fossil fuels and nuclear known as non renewable source of energy. The consumption of these non renewable sources of energy, impacts on the environment by spoiling the fauna, flora and the climatic changes. To face this issue of pollution, the development of new cleaning non-polluting sources of energy must be explored. Fortunately, thanks to the renewable energies, our expectations can be met (Rahul et al., 2014). This type of energy has revolutionized the energy sector worldwide. It can be subdivided into several branches such as solar energy, wind, geothermal energy just to name a few (Nottom et al., 1996). The above-mentioned energies come mainly from solar energy (Sanjeev et al., 2016) which is a second form of energy used by human being after the muscular energy. In addition, the energy coming from the sun is very important and presents a solar potential of about $1340 \mathrm{~W} / \mathrm{m}^{2}$. This potential can be directly transformed into electricity thanks to the PV cells and into thermal energy through the solar collector depending of needing. However, in the view of solving the need of electrical energy in isolated area, people used more than more PV panels based on photovoltaic conversion. Since the pioneering work of Becquerel (1939) showing that photovoltaic effect results from the interaction between a photon (quantum of light) and the matter (electron) which produces current and electric voltage, this concept has been intensively studied. Latter Einstein (1917) explained the photovoltaic effect using the basic principle of quantum mechanics. Depending on the current or voltage sought, solar cells can be grouped in series, parallel, and compound to form a PV module (Emmanuel, 2006). Despite the conversion of the solar radiation into electricity thank to a PV cell, its electrical efficiency is still very low due to the factors that affect negatively its dynamics. It is the reason why since the past decades many researches are continued to be done aiming to optimize its electrical performances and maintain it in operating temperature. This can be explained by many research works. Indeed, Siyu et al. (2016) studied the local effects of inhomogeneity of a silicon solar cell. They showed that, the inhomogeneity causes the decrement of the open circuit voltage of a PV cell while the short circuit current increases. Osueke (2015) optimized the parameters of the solar cells through an experiment based on the angle of tilt, the angle of azimut. He showed that the angle of tilt increases with the electrical efficiency. Following the same goal, Mohamed et al. (2008) conducted studies on an exponential model of a series resistance. Their work revealed that this profile of series resistance optimizes the electrical efficiency of a solar cell. Still in the line to improve the performances of a solar cell, Shaharim et al. (2011) established that the accumulation of dust on the surface of a solar cell causes the drop of its electrical parameters of about $50 \%$. On the order hand, Garg et al. (1973) demonstrated that when a PV module is inclined under an angle of 45 degrees, its transmission coefficient and its performance both decrease. In 2010, Nottom et al. showed that when a PV module is shaded, its electrical performances decrease. Moreover, Mohammad et al. (2016) and Xu et al. (2014) established that, when a solar cell is exposed to the sun, about $9-12 \%$ of the solar radiation is converted into electricity. The rest is absorbed by the solar cell, leading to the growth of its operating temperature and therefore to the reduction of its electrical efficiency about $0.45 \%$ per degree Celsius (Davud et al., 2013; Gao et al., 2010; Kalogirou et al., 2006; Latifa et al., 2014; Skoplaki et al., 2009). Furthermore, as the nature is essentially nonlinear, Dongo et al. (2014) worked on the effects of a thermal nonlinear series resistance, based on an electrical model of a solar cell. They showed 
that the nonlinear character of the series resistance optimizes of about $3.6 \%$ the power of a PV cell. These previous mentioned works traduce the great desire to deeply master the dynamics of the PV cell in order to propose new approaches that assure their stabilities and improve their yield.

Highly motivated by the nonlinearity like Dongo et al. (2014) and the amount of energy dissipated in the PV module, we plan to study theoretically the effect of a nonlinear absorption coefficient of a PV module. This coefficient is expressed as a quadratic function of the temperature across it, in replacement of the constant absorption coefficient commonly used in the scientific literature. To achieve our goal, the structure of this paper is organized as follows. In section 2, we present the different parts of a PV panel which lead to the mathematical formulations thanks to the energy balance. In section 3, simulations of the mathematical expressions are made exploiting the MATLAB environment. Comparison and discussion of the results obtained are carried out in the cases of constant and nonlinear absorption coefficients of a solar cell, ended by discussions and concluding remarks.

\section{Mathematical formulation}

\subsection{Description of a PV panel}

A PV panel consists of several layers like glass, active zone known as PV module, Teldar and EVA as shown on figure 1. However, in practice the depth and heat capacities of Teldar and EVA are very small justifying why these layers are neglected in various studies. Therefore, the simplified PV panel is made of two layers as drawn on figure 2. On this figure, the first layer is the glass that physically protects the solar cell against the projectiles capable to destroy the PV panel. Known as the electronic part, the second layer is the PV module consisting of an association of identical solar cells in which the photogeneration, the diffusion and the recombination process of minority carriers take place.

Within our investigations, the sky is considered as a black body at constant temperature. It is also assumed that losses at the front and back surface of the PV panel are identical, and the effect of the dust at the front surface of the PV panel is negligible. We also consider that the temperature of each layer varies slowly with time. Then, the time derivative in the energy balance is neglected. We also admit that our searches are conducted in the steady state, and that the solar radiation is considered uniform on the PV panel. Based on the above assumptions, the temperature, thermodynamics constants as well as the optical properties are also uniform in each layer of the PV panel (Emmanuel, 2006).

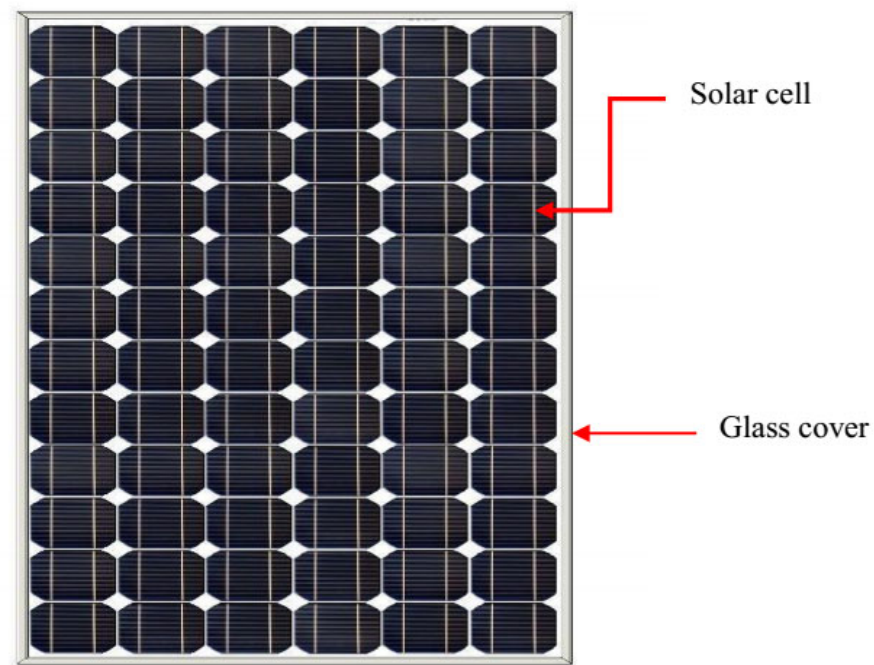

Figure 1: General view of a PV panel. 


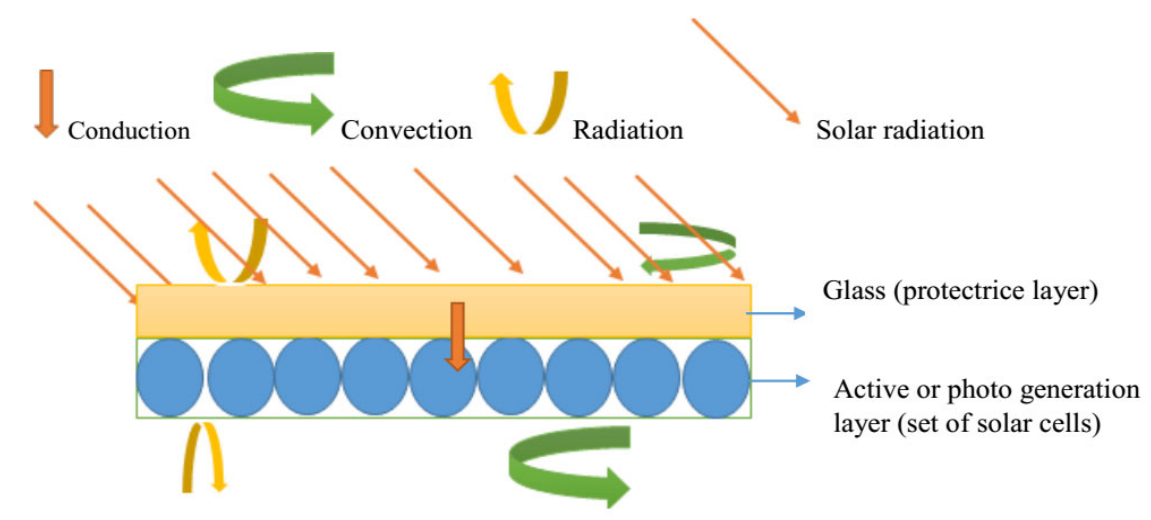

Figure 2 : Presentation of the Cross Section of a PV Panel with the various heat transfers

\subsection{Energy balance of glass cover}

Glass is the first layer of a photovoltaic panel. Owing to Tiwari et al. (2006), the energy balance through this layer is given by:

$$
\alpha_{g} G(t)=U_{g a}\left(T_{g}-T_{a}\right)+U_{g c}\left(T_{g}-T_{c}\right)
$$

in which the quantity $T_{c}$ defines the temperature of the PV module while $T_{g}$ and $\alpha_{g}$ designate the temperature and the absorption coefficient of the glass cover, respectively. Based on Eq. (1), one derives the temperature of the glass defined as

$$
T_{g}=\frac{\alpha_{g} G(t)+U_{g a} T_{a}+U_{g c} T_{c}}{U_{g c}+U_{g a}}
$$

where $U_{g c}=1 /\left(\left(L_{g} / K_{g}\right)+\left(L_{c} / K_{c}\right)\right) \quad$ and $\quad U_{g a}=1 /\left(h_{c o n v}+h_{r a d}\right)$

In the previous relations, the parameter $U_{g c}$ stands for the coefficient of thermal loss between the glass cover and the active zone whereas $U_{g a}$ designates the coefficient of thermal loss between the glass cover and the ambient milieu.

In (2), the parameter $G(t)$ stands for the solar radiation intensity during the whole day. In scientific reviews, there are several models of solar radiation intensity existing such as Adnot (Ionel et al., 2015), Karsten (Kasten et al., 1989), Haurwitz (Ionel et al., 2015) and Empirical model (Paulescu et al., 2003). It has clearly appeared that the model of Adnot is the best since it describes the dynamics of the solar radiation nearest the reality. Then, this model is considered during our investigations and is expressed as follows (Ionel et al., 2015):

$$
G=951.39 \sin \left(\alpha_{s}\right)^{1.15}
$$

in which $\sin \left(\alpha_{s}\right)=\sin (\delta) \sin (\varphi)+\cos (\delta) \cos (\varphi) \cos (w)$, wherein $\varphi$ is the latitude of the considered location and $\delta$ represents the declination of the Earth. The parameter $w$ defines the position of the sun in the sky at any time and is expressed as follows:

$$
w=15 \times(12-T)
$$

where $T$ represents the time.

\subsection{Energy balance in the PV module}

In the literature, nonlinearity generates new harmonics in a given dynamical system, offers a good advantage and is used to produce chaotic behavior, masking of the information. Beyond these applications, nonlinearity offers very often advantages in providing energy in a system (Dongo et al., 2014; Pelap et al., 2016). Motivated by this positive effect, we replace the constant absorption coefficient of the PV module by a nonlinear absorption coefficient expressed as a quadratic function of the temperature, since the variation of this coefficient due to the joule effect is usually neglected:

$$
\alpha_{c}=\alpha_{c 0}\left(1+\beta T_{c}+\theta T_{c}^{2}\right)
$$

wherein $\theta$ is the quadratic coefficient consequently chosen. The quantities $\alpha_{c 0}, T_{c}$ and $\beta$ are, respectively, the normalized thermal absorption coefficient, the temperature of the PV module and the coefficient of thermal linear expansion of the material (Dongo et al., 2014; Fouad et al., 2012).

In the active zone of the PV panel known as PV module, many processes take place like photo generation, 
diffusion and recombination of minority carriers. The rise of the temperature of the PV panel is mainly linked to this zone. According to Tiwari et al. (2006), the energy balance at this layer is given by

$$
U_{g c}\left(T_{g}-T_{c}\right)+\tau_{g} \alpha_{c} \beta_{c} G(t)=h_{c p}\left(T_{c}-T_{a}\right)+\tau_{g} \alpha_{c} \beta_{c} \eta_{c} G(t)
$$

With $h_{c p}=\frac{1}{\frac{L_{c}}{K_{c}}+h_{\text {conv }}} ; \quad h_{\text {rad }}=\frac{\varepsilon_{g} \sigma\left(T_{g}^{2}+T_{s k y}^{2}\right)\left(T_{g}+T_{s k y}\right)\left(T_{g}-T_{s k y}\right)}{\left(T_{g}-T_{a m}\right)}$ and $h_{c o n v}=5.7+3.8 \mathrm{~V}$

In equation (6), $V$ designates the wind speed, $h_{\text {conv }}$ is the convective transfer coefficient, $h_{\text {rad }}$ represents the radiative transfer coefficient and $h_{c p}$ defines the coefficient of thermal loss between the PV module and the ambient milieu. The quantities $\tau_{g}, \alpha_{c}, \eta_{c}, L_{c}, K_{c}, T_{a m}, T_{s k y}$ and $\beta_{c}$ refer, respectively, to the transmission coefficient of the glass, the absorption coefficient, the electrical efficiency, the thickness, the thermal conductivity of PV module, the ambient temperature, the sky temperature and the packing factors designating the fraction of the PV module covert by the glass layer. Substitution of expressions (5) into Eq. (6) leads to the following relation:

$$
A A T_{c}^{2}+B B T_{c}+C C=0
$$

in which we have put:

$$
\begin{gathered}
A A=-\tau_{g} \alpha_{c 0} \beta_{c} \theta\left(1-\eta_{c}\right) G(t) ; B B=h_{c o n v}+U_{1}-\tau_{g} \alpha_{c 0} \beta_{c} \beta\left(1-\eta_{c}\right) G(t) \\
C C=-\left(\tau_{g} \alpha_{c 0} G(t)-\eta_{c} \tau_{g} \alpha_{c 0} G(t)+P F_{1} \alpha_{g} G(t)+U_{1} T_{a m}+h_{c o n v} T_{a m}\right) \\
P F_{1}=U_{g c} /\left(U_{g c}+U_{g a}\right) \quad \text { and } \quad U_{1}=U_{g a} U_{g c} /\left(U_{g c}+U_{g a}\right)
\end{gathered}
$$

with

Resolution of Eq. (7) yields two mathematical solutions for the temperature for the PV module and the accepted physical solution is defined below.

$$
T_{c}=\frac{-B B+\sqrt{B B^{2}-4 \cdot A A \cdot C C}}{2 \times A A}
$$

Fortunately, the MATLAB environment offers the possibility to forward the study and examine the contribution of the nonlinear absorption coefficient on the dynamics of the PV module

\section{Results and Discussion}

\subsection{Effect of a nonlinear absorption coefficient on the glass cover}

In this subsection, we conduct numerical simulations of the mathematical expression (2) of the temperature of the glass cover in the MATLAB environment. These investigations of the thermal behavior of the glass cover are carried out for both constant and nonlinear absorption coefficients as shown on figure 3 . The curves are obtained for various values of quadratic coefficient $\theta$ with the following system parameters: $\alpha_{g}=0.05, \tau_{g}=0.95$ and $T_{a m}=298.15 \mathrm{~K}$. We treat the case of the silicon PV module materialized by the values of $\alpha_{c 0}=0.95$ and $\beta=2.6 \times 10^{-6} K^{-1}$. For the constant absorption coefficient (in black color on figure 3 ), we note that the profile of the temperature of the glass cover follows that of the solar irradiation. It could be also noted that the maximum temperature is around $345^{\circ} \mathrm{K}$ (i.e., $70^{\circ} \mathrm{C}$ ) at the midday. However, for various values of the nonlinear absorption coefficient $(\theta)$, we remark that the shape of the obtained curves (in color on figure 3 ) does not change. It also appears that the value of the maximum temperature decreases as the parameter $\theta$ grows. According to these results, the nonlinear character of the absorption coefficient plays the role of a cooling system for the glass cover. 


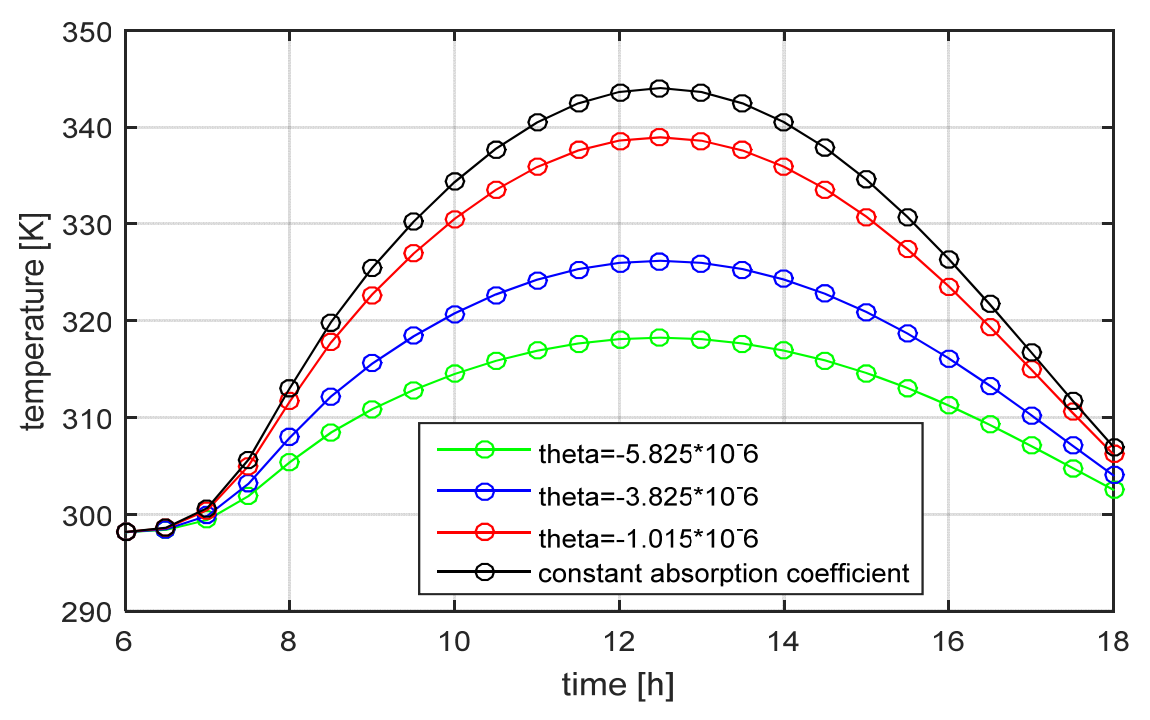

Figure 3: Glass cover temperature versus time for constant and nonlinear absorption coefficient made with the system parameters: $\alpha_{c 0}=0.95, \beta=2.6 \times 10^{-6} K^{-1}, \alpha_{g}=0.05, \tau_{g}=0.95, T_{a m}=298.15 K$ The presence of the nonlinear absorption coefficient of the PV module reduces the temperature of the glass cover.

\subsection{Effect of a nonlinear absorption coefficient on a PV module}

Figures 4-5 show variations of both the temperature and the electrical efficiency of the PV module using the parameters and conditions of figure 3 . Numerical simulations of the mathematical expression (8) of the temperature of the PV module are made under MATLAB environment. In the case of constant absorption coefficient plotted in black color on figure 4, we observe that the maximum temperature of the PV module is closed to 70 degrees, but remains slightly higher than the temperature of the glass cover obtained in the same conditions (Swapnil et al., 2009). Indeed, this difference of temperature is linked to the smallness of the heat capacity of the glass compared to that of the active zone known as PV module. Furthermore, several processes like recombination effect take place in a PV module. In fact, this recombination effect contributes also to the rise of the temperature of that layer thanks to the joule effect (Ibrahima et al., 2016).

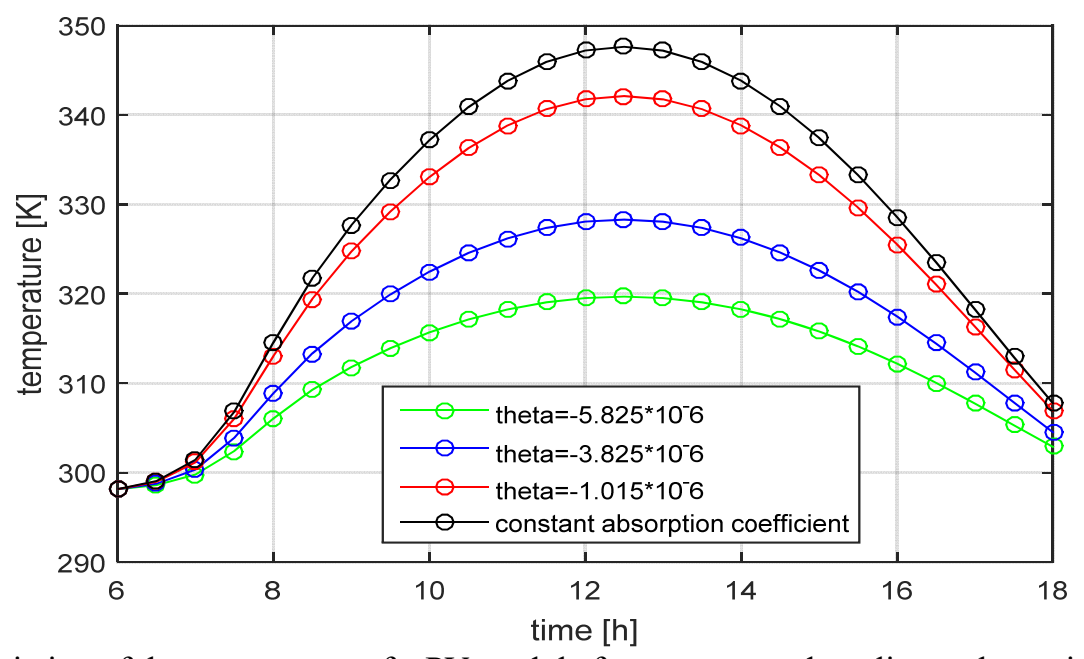

Figure 4: Variation of the temperature of a PV module for constant and nonlinear absorption coefficient obtained with same system parameters as in figure 3. The presence of the nonlinear absorption coefficient of the PV module reduces both the recombination effect in that zone and also its temperature. 


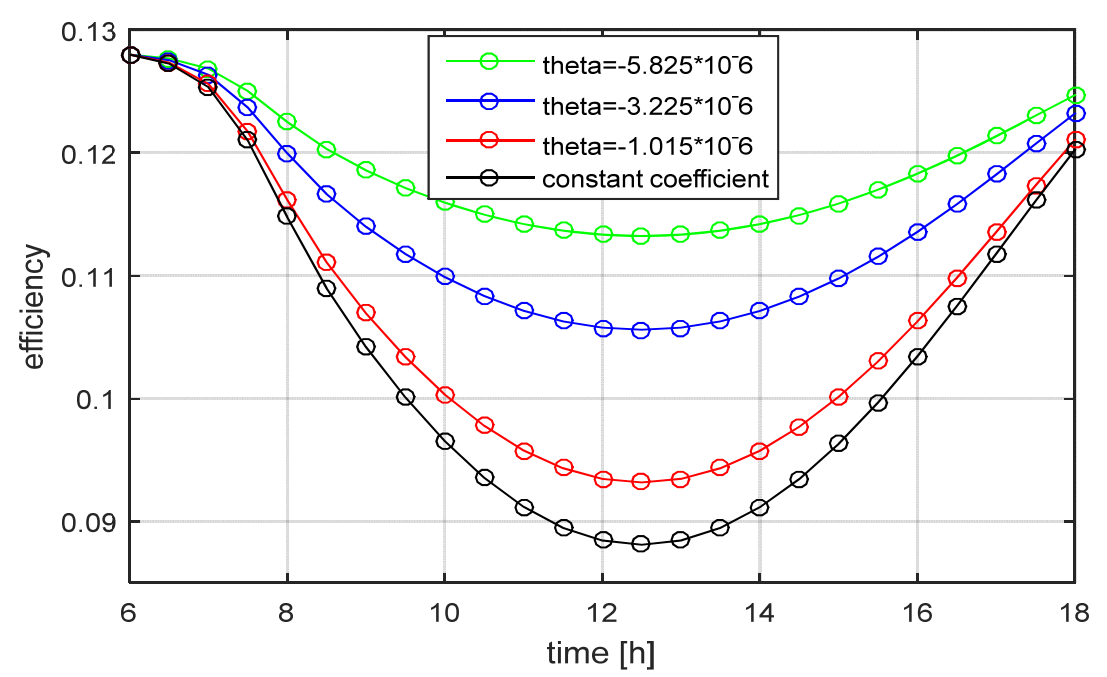

Figure 5: Evolution of the electrical efficiency of a PV module for constant and nonlinear absorption coefficient. The maximum electrical efficiency of the PV panel increases when the quadratic coefficient $\theta$ decreases.

However, by taking into account the nonlinear behavior of the absorption coefficient of this layer, we note that the temperature of the PV module retains the shape of the solar radiation. Moreover, its maximum temperature decreases and reaches the minimum for $\theta=-5.825 \times 10^{-6} K^{-2}$ (Table 1). Indeed, this coefficient $\theta$ impacts positively on the thermal behavior of the PV module by reducing both the recombination effect taking place as well as its operating temperature. In addition, the curves of figure 5 display that the electrical efficiency of the PV module grows as $\theta$ diminishes (Table1). These results are in good accordance with those established by Pelap et al. (2016) in which they proved the useful of the nonlinearity in the solar cell domain. Thus, the nonlinear absorption coefficient reduces both the temperature and the recombination effect of a PV module and therefore increases it electrical efficiency.

Table 1. Numerical data collected on each layer of the PV panel at the turning point for various values of the quadratic coefficient $\theta$.

\begin{tabular}{|l|c|c|c|c|}
\hline \multicolumn{1}{|c|}{ Quantity Identification } & \multicolumn{3}{c|}{ Corresponding Value } \\
\hline Quadratic coefficient $\theta \times 10^{-6}\left(K^{-2}\right)$ & -5.825 & -3.825 & -1.015 & 0 \\
\hline Temperature of the glass $T_{g}($ Kelvin degree) & 319 & 327 & 339 & 342 \\
\hline Temperature of the PV module $T_{c}$ (Kelvin degree) & 320 & 329 & 341 & 347 \\
\hline Electrical efficiency (\%) & 11.32 & 10.56 & 9.31 & 8.81 \\
\hline
\end{tabular}

\section{Conclusion}

In this paper, we have analyzed theoretically the effects of a thermal nonlinear absorption coefficient on the dynamics of a PV panel. This coefficient is expressed as a quadratic function of the temperature of the PV module known as the electronics active part of a PV panel. Based on the energy balance equation, the mathematical expressions of the evolution of the temperatures in at each layer of the PV panel are determined. Numerical investigations carried out thanks to the MATLAB environment have exhibited some new features. Indeed, our founding have revealed that the nonlinear character of the absorption coefficient induces the cooling of the PV panel, reduces the recombination effects and therefore increases the electrical efficiency of the PV panel, for the nonlinear thermal coefficient $\theta$ chosen between $-5.825 \times 10^{-6} \mathrm{~K}^{-2}$ and $-1.015 \times 10^{-6} \mathrm{~K}^{-2}$. The above results offer to the manufacturers of PV panel a new trick to improve the electrical efficiency of the PV modules and reduce both their operating temperatures and recombination effects. For future works, we plan to study other nonlinear aspects of a PV panel.

\section{References}

Becquerel, E. (1839). Mémoire sur les effets électriques produits sous l'influence des rayons solaires. Comptes Rendus, 9, 561-567.

Davud, M.T., Madatov, R.., Daryush, N. (2013). The Effect of temperature on electrical parameters of solar cells. International Journal of Advanced Research in Electrical, Electronics and Instrumentation Engineering, 2 , 6404-6407.

Dongo, P.D., Kapim, A.D., Pelap, F.B. (2014). Effects of a thermal nonlinear resistance on the power output of the PV Cell. Journal of Energy Technologies and Policy, 4, 101-111. 
Einstein, A. (1917). On the Quantum Theory of the Radiation. Phyikalishe Zeitschrift, 18, 1-15.

Emmanuel, A. (2006). Etude du comportement thermique de modules photovoltaïques en vue de la prédiction de leur production sur site. Thèse de doctorat. Université de PROVENCE : Institut des Systèmes Thermiques Industriels.

Fouad, K., Ali, B., Patrick, P. (2012). Modelling of thermal behavior N-doped silicon resistor. Journal of Sensor Technology, 2, 132-137.

Gao, Y., Huang, H., Su, Y., Riffat, S.B. (2010). A parametric study of characteristics of concentrating PV modules. International Journal of Low-Carbon Technology, 5, 7-62.

Garg, H.P. (1973). Effect of dirt on transparent covers in flat-plate solar energy collectors. Solar Energy, 15, 299302.

Ibrahima, D., Ibrahima, L., Mamadou, W., Marcel, S.D., Senghane, M. (2016). Temperature effect on capacitance of a silicon solar cell under constant white biased light. World Journal of Condensed Matter Physics, 6, 261268

Ionel, L.A., Cornelia, A.B., Sonia, D. (2015). Estimating solar irradiation absorbed by photovoltaic panels with low concentration located in Craiova, Romania. Sustainability, 7, 2644-2661.

Kalogirou, S.A., Tripanagnostopoulos, Y. (2006). Hybrid PV/T solar systems for domestic hot water and electricity production. Energy Conversion and Management, 47, 3368-3382.

Kasten, F., Young, A. (1989). Revised optical air mass tables and approximation formula. Applied Optics, 22 , 4735-4738.

Latifa, S., Mohammed, B. (2014). Effect of ambient conditions on thermal properties of photovoltaic cells: Crystalline and amorphous silicon. International Journal of Innovative Research in Science, Engineering and Technology, 3, 17815-17821.

Mohamed, O.Y., Mahmoud, O., Youm, I. (2008). Etude et modélisation d'un générateur photovoltaïque. Revue des Energies Renouvelables, 11, 473-483.

Mohammad, S., Mohamed, G. (2016). Performance assessment of integrated PV/T and solid desiccant airconditioning systems for cooling buildings using Maisotsenko cooling cycle. Solar Energy, 127, 79-95.

Nottom, G., Caluianu, I., Colda, I., Caluianu, S. (2010). Influence d'un ombrage partiel sur la production electrique d'un module photovoltaïque en silicium monocristallin. Revues des énergies renouvelables, 1, 49-62.

Notton, G., Musseli, M., Poggi, P., Louche, A. (1996). Autonomous photovoltaic systems influences of some parameters on the sizing: simulation time step, input and output power profil, Renewable Energy, 1, 5569.

Osueke (2015). Design of a solar power system for optimal output. International Journal of Advanced Engineering Sciences and Technologies, 11, 233-237

Paulescu, M., Schlett, Z. (2003). Performances assessment of global solar irradiation models under Romanian climate. Renew Energy, 5, 767-777.

Pelap, F.B., Dongo, P.D., Kapim, A.D. (2016). Optimization of the characteristics of the PV cells using nonlinear electronic components. Sustainable Energy Technologies and Assessments, 16, 84-92.

Rahul, S.R., Hariharam, R. (2014). Performance study of solar photovoltaic thermal collector integrated with cooling system. International Journal of Emerging Engineering Research and Technology, 2, 132-145.

Sanjeev, J., Soni, M.S., Nikhil, G. (2016). Historical and recent development of concentrating of photovoltaic cooling technologies. Renewable and Sustainable Energy Review, 60, 41-59.

Shaharin, A.S., Haizatul, H.H., Nik,S.H., Nik, L., Mohd, S.I.R. (2011). Effects of dust on the performance of PV panels. International Journal of Mechanical and Mechatronics Engineering, 5, 2028-2033.

Siyu, G., Armin, G., Marius, P. (2016). Investigating local inhomogeneity effects of silicon wafer solar cell by circuit modeling. Energy Procedia, 33, 7-10.

Skoplaki, E., Palyvos, J.A. (2009). On the temperature dependence of photovoltaic module electrical performance. A review of efficiency/power correlations Sol Energy, 83, 614-624.

Tiwari, A., Sodha, M.S (2006). Performance evaluation of solar PV/T system: An experimental validation. Solar Energy, 80, 751-759.

Tiwari, A., Sodha, M.S., Avinash, C., Joshi, J.C. (2006). Performance evaluation of photovoltaic thermal solar air collector for composite climate of India. Solar Energy Materials \& Solar Cells, 90,175-189.

Tiwari, A., Swapnil, D., Solanki, S.C. (2009). Energy and energy analysis of PV/T air collectors connected in series. Energy and Buildings, 41, 863-870.

$\mathrm{Xu}, \mathrm{Z}$., Kleinstreuer, C. (2014). Concentration photovoltaic-thermal energy co-generation system using nanofluids for cooling and heating. Energy Conversion and Management, 87, 504-512. 